\title{
No early benefits of adjunct therapy with tolvaptan for acute heart failure
}

Tolvaptan, a vasopressin 2-receptor antagonist that induces loss of free water, has been proposed as a good candidate for adjunct therapy to improve congestion and symptoms in patients with acute heart failure. Two clinical trials now show that tolvaptan as an adjunct to loop diuretics, despite inducing early weight and fluid loss, is not associated with early (1 day) improvements in congestion and dyspnoea in patients with decompensated heart failure. However, at day 3 the reduction in dyspnoea was significantly greater with tolvaptan than with placebo in the SECRET of CHF trial, with a similar trend found in the TACTICS-HF trial. "There seems to be a temporal separation between differential fluid loss and dyspnoea improvement," says Marvin A. Konstam, lead investigator of the SECRET of CHF study.

Both trials focused on early randomization (within $24 \mathrm{~h}$ of hospitalization in TACTICS-HF and $36 \mathrm{~h}$ in SECRET of CHF), on the basis of previous studies showing that early tolvaptan treatment was more likely to be associated with improvements in symptoms. In addition, the SECRET of CHF trial focused on patients who were most likely to respond to tolvaptan (those with hyponatraemia, renal dysfunction, or diuretic resistance), and both trials used standardized Likert scores for dyspnoea assessment. Nevertheless, despite the improved design neither of the trials met the primary end point.

Interestingly, a subgroup analysis in the SECRET of CHF trial showed that patients without signs of right heart failure had a more rapid improvement in dyspnoea scores. "Going forward, it will be essential to stratify patients according to signs of right heart failure, which seem to be associated with significant differences in clinical response," remarks Konstam.

Irene Fernández-Ruiz

ORIGINAL ARTICLES Konstam, M. A. et al. Short-term effects of tolvaptan in patients with acute heart failure and volume overload. J. Am. Coll. Cardiol. 69, 1409-1419 (2017)|

Felker, G. M. et al. Efficacy and safety of tolvaptan in patients hospitalized with acute heart failure. J. Am. Coll. Cardiol. 69. 1399-1406 (2017) 